\title{
Repairing the efficiency loss due to varying cluster sizes in two-level two-armed randomized trials with heterogeneous clustering
}

Citation for published version (APA):

Candel, M. J. J. M., \& Van Breukelen, G. J. P. (2016). Repairing the efficiency loss due to varying cluster sizes in two-level two-armed randomized trials with heterogeneous clustering. Statistics in Medicine, 35(12), 2000-2015. https://doi.org/10.1002/sim.6851

Document status and date:

Published: 30/05/2016

DOI:

10.1002/sim.6851

Document Version:

Publisher's PDF, also known as Version of record

Document license:

Taverne

Please check the document version of this publication:

- A submitted manuscript is the version of the article upon submission and before peer-review. There can be important differences between the submitted version and the official published version of record.

People interested in the research are advised to contact the author for the final version of the publication, or visit the DOI to the publisher's website.

- The final author version and the galley proof are versions of the publication after peer review.

- The final published version features the final layout of the paper including the volume, issue and page numbers.

Link to publication

\footnotetext{
General rights rights.

- You may freely distribute the URL identifying the publication in the public portal. please follow below link for the End User Agreement:

www.umlib.nl/taverne-license

Take down policy

If you believe that this document breaches copyright please contact us at:

repository@maastrichtuniversity.nl

providing details and we will investigate your claim.
}

Copyright and moral rights for the publications made accessible in the public portal are retained by the authors and/or other copyright owners and it is a condition of accessing publications that users recognise and abide by the legal requirements associated with these

- Users may download and print one copy of any publication from the public portal for the purpose of private study or research.

- You may not further distribute the material or use it for any profit-making activity or commercial gain

If the publication is distributed under the terms of Article $25 \mathrm{fa}$ of the Dutch Copyright Act, indicated by the "Taverne" license above, 


\title{
Repairing the efficiency loss due to varying cluster sizes in two-level two- armed randomized trials with heterogeneous clustering
}

\author{
Math J. J. M. Candel ${ }^{* \dagger \dagger}$ and Gerard J. P. Van Breukelen
}

\begin{abstract}
In two-armed trials with clustered observations the arms may differ in terms of (i) the intraclass correlation, (ii) the outcome variance, (iii) the average cluster size, and (iv) the number of clusters. For a linear mixed model analysis of the treatment effect, this paper examines the expected efficiency loss due to varying cluster sizes based upon the asymptotic relative efficiency of varying versus constant cluster sizes. Simple, but nearly cost-optimal, correction factors are derived for the numbers of clusters to repair this efficiency loss. In an extensive Monte Carlo simulation, the accuracy of the asymptotic relative efficiency and its Taylor approximation are examined for small sample sizes. Practical guidelines are derived to correct the numbers of clusters calculated under constant cluster sizes (within each treatment) when planning a study. Because of the variety of simulation conditions, these guidelines can be considered conservative but safe in many realistic situations. Copyright (C) 2016 John Wiley \& Sons, Ltd.
\end{abstract}

Keywords: asymptotic relative efficiency; cluster randomized trials; individually randomized group treatment; therapist effects; varying cluster sizes

\section{Introduction}

Trials evaluating the effect of a treatment are often characterized by observations that are correlated within clusters. A prototypical example is cluster randomized trials [1], where organizational units, such as general practices, instead of individuals, such as patients, are assigned to one of several treatment conditions, and all individuals within the same unit receive the same treatment, such as a certain medication regime. Clustering of observations may also occur when individuals are the units of assignment, but the treatment itself induces clustering, for instance, when treatments are delivered in group sessions as in psychotherapy [2]. In such individually randomized group treatment trials [3-5], interactions between persons within a group may lead to their outcomes being correlated. An example is a study by Baldwin et al. [4], in which high school and university students expressing body image concerns either received a group-administered dissonance intervention or a group-administered healthy weight management program. Here, clustering was found on the score on the Revised Ideal Body Stereotype Scale, a self-report measure of internalization of the thin beauty ideal. Clustering effects due to treatment may even occur if the treatment is given on an individual basis. Patients that are treated by the same therapist or, more generally, the same care provider, will be treated in a more similar way than patients treated by different therapists. Such therapist effects may also be a source of observations becoming correlated within groups $[2,3,6]$.

For two-armed designs with clusters of correlated observations in each arm, formulas for sample size calculation have been derived $[1,7,8]$, assuming the arms have the same outcome variance, the same intraclass correlation, the same number of clusters and the same cluster size. However, the outcome variance is likely to be heterogeneous across treatments if they have any effect on the outcome variable [9],

Department of Methodology and Statistics, School for Public Health and Primary Care CAPHRI, Maastricht University Maastricht, The Netherlands

*Correspondence to: Math J.J.M. Candel, Department of Methodology and Statistics, Maastricht University, P.O. Box 616, 6200 MD Maastricht, The Netherlands.

${ }^{\dagger}$ E-mail: math.candel@maastrichtuniversity.nl 
and treatment arms with different numbers of clusters and cluster sizes may result from minimization of the total research costs when the cost per cluster respectively per person differs between treatment arms $[8,10]$ or simply due to the nature of the treatments, for instance in comparing two forms of group psychotherapy which differ in the maximum feasible group size. Such heterogeneity of variance and sample size between treatment arms must be taken into account in the model for data analysis and consequently also in the sample size calculation. For a two-sample $t$-test, it is well known that neglecting the heterogeneity in outcome variance between treatment arms may lead to biased tests, especially when the group sizes differ between treatments arms and the extent of heterogeneity is large (e.g. [11,12]). Because a mixed model analysis of two-armed cluster randomized trials is equivalent to a two sample $t$-test on cluster means if clusters are equally sized within arms [13], similar biases will occur in such a mixed model analysis if the variance parameters and the number of clusters differ between treatment arms, whereas the model for analysis assumes homogeneity of variance.

Formulas for sample size calculation that accommodate heterogeneity of variance and sample size between arms in the context of a linear mixed model analysis have been presented by Candel et al. [13]. However, these formulas assume that cluster sizes are equal within (but not between) treatment arms, whereas varying cluster sizes is the more common case. Therefore, this paper will examine the effect of cluster size variation within arms on design efficiency in the presence of heterogeneity of variance and sample size between arms. It will also examine how to repair the efficiency loss incurred by cluster size variation in a cost-efficient way when planning sample sizes.

The efficiency loss will be studied for a mixed effects approach to analyzing the data. An alternative is the generalized estimating approach, which may be more robust to misspecifying the random effects part of the model, provided the sample size is large [14]. However, for small numbers of clusters generalized estimating equations is known to lead to biased estimates of the standard errors of the fixed effects estimators, yielding inflated type I error rates and undercoverage of confidence intervals $[15,16]$. For small numbers of clusters, sometimes an analysis on cluster summary measures, notably cluster means, is advised (e.g. [17]). Unequal cluster sizes imply heteroscedasticity of cluster means, however, which invalidates the unpaired $t$-test on cluster means. In this paper, we will therefore consider a mixed effects approach.

The efficiency loss due to varying cluster sizes within arms will be derived for the asymptotic case and checked for finite samples in an extensive Monte Carlo simulation study, considering both maximum likelihood (ML) and restricted maximum likelihood estimation (REML). A simple Taylor approximation of the asymptotic efficiency loss, based on the coefficient of variation of cluster sizes, will be derived and checked against the simulation results. This approximation may be very practical in taking care of the efficiency loss when planning a study. For the case of homogeneity of intraclass correlation, outcome variance, number of clusters, average cluster size and cluster size distribution between arms, this Taylor approximation is known to be much more precise than a commonly used approximation based on cluster size weighting [18-20]. We will also briefly examine to what extent this is true when there is between-arm heterogeneity in the variance parameters as well as in the number of clusters and the average cluster sizes. Furthermore, because of the small sample characteristics of individually randomized group treatment trials and trials with therapist effects, the accuracy of the asymptotic approximation and the Taylor approximation is studied for cluster sizes smaller than considered before [21,22].

The paper is structured as follows. Section 2 presents the linear mixed effects model for two-armed trials, allowing for different intraclass correlations, different outcome variances, different cluster numbers and different cluster sizes in these arms. Section 3 then presents the asymptotic relative efficiency (ARE) of unequal versus equal cluster sizes within arms, and Section 4 presents a Taylor approximation to this ARE that can be used to compensate for the expected efficiency loss because of cluster size variation in planning a study. Section 5 discusses a Monte Carlo study evaluating the accuracy of the ARE and its Taylor approximation for various cluster size distributions with realistic sample sizes. Section 6 illustrates how to apply the results in planning the sample sizes for a trial, whereas Section 7 summarizes the implications of the present study.

\section{Specification of the linear mixed effects model}

In the treatment and control condition, we have respectively $K_{t}$ and $K_{c}$ clusters (such as $K_{t}$ and $K_{c}$ therapy groups). In cluster $j\left(j=1, \ldots, K_{t}\right)$ of the treatment condition, there are $m_{j}^{t}$ persons, and in cluster $j\left(j=K_{t}+1, \ldots, K_{t}+K_{c}\right)$ of the control condition, there are $m_{j}^{c}$ persons, the total number of persons thus 
amounting to $N=\sum_{j=1}^{K_{t}} m_{j}^{t}+\sum_{j=K_{t}+1}^{K_{t}+K_{c}} m_{j}^{c}$. The dependent variable is a quantitative outcome, denoted as $y_{i j}$ for person $i$ in cluster $j\left(j=1, \ldots, K_{t}+K_{c}\right)$. If in each condition $y_{i j}$ is (approximately) normally distributed, the following linear mixed effects model is an adequate tool for data analysis (cf. Roberts et al. [2]; Walwyn et al. [6]):

$$
y_{i j}=\beta_{0}+\left(\beta_{1}+u_{j}+\delta_{i j}\right) \operatorname{Int}_{i j}+\left(v_{j}+\varepsilon_{i j}\right)\left(1-\operatorname{Int}_{i j}\right),
$$

where $I n t_{i j}$ denotes the treatment condition for person $i$ in cluster $j$, which is coded as 1 for persons in the treatment condition and coded 0 for persons in the control condition. With this coding scheme, $\beta_{0}$ represents the mean score of the control condition, and $\beta_{1}$ represents the treatment effect, such as the mean outcome difference between cognitive behavioural therapy and psychodynamic interpersonal psychotherapy [5]. Commonly, $\beta_{1}$ is the parameter of primary interest. The terms $u_{j}$ and $v_{j}$ represent the random cluster effect in the treatment and control condition, respectively, whereas $\delta_{i j}$ and $\varepsilon_{i j}$ represent a random person effect in these conditions, respectively. The effects $u_{j}, v_{j}, \delta_{i j}$ and $\varepsilon_{i j}$ are assumed to be independently normally distributed with variances $\sigma_{u}^{2}$, $\sigma_{v}^{2}, \sigma_{\delta}^{2}$ and $\sigma_{\varepsilon}^{2}$. The total outcome variances for the treatment and control arm are $\sigma_{y_{t}}^{2}=\sigma_{u}^{2}+\sigma_{\delta}^{2}$ and $\sigma_{y_{c}}^{2}=\sigma_{v}^{2}+\sigma_{\varepsilon}^{2}$.

The intraclass correlation measures the dependency among observations for members within the same cluster. For the model in Eqn (1) the intraclass correlations are $\rho_{t}=\sigma_{u}^{2} /\left(\sigma_{u}^{2}+\sigma_{\delta}^{2}\right)=\sigma_{u}^{2} / \sigma_{y_{t}}^{2}$ for the treatment arm and $\rho_{c}=\sigma_{v}^{2} /\left(\sigma_{v}^{2}+\sigma_{\varepsilon}^{2}\right)=\sigma_{v}^{2} / \sigma_{y_{c}}^{2}$ for the control arm. The correlation between two randomly drawn persons from two different clusters is zero.

\section{Asymptotic relative efficiency}

Let $\xi_{\text {equal }}$ and $\xi_{\text {unequal }}$ denote two designs with the same numbers of clusters $K_{t}, K_{c}$ and the same average cluster sizes $\bar{m}^{t}, \bar{m}^{c}$ for the two treatment arms, but differing in that the cluster sizes within each treatment arm are equal in $\xi_{\text {equal }}$ but vary in $\xi_{\text {unequal }}$. Let $\operatorname{var}\left(\hat{\beta}_{1} \mid \xi_{\text {equal }}\right)$ and $\operatorname{var}\left(\hat{\beta}_{1} \mid \xi_{\text {unequal }}\right)$ denote the corresponding asymptotic variances of the treatment effect estimator. The asymptotic relative efficiency (ARE) of a design with unequal versus a design with equal cluster sizes for the estimator of the treatment effect, $R E\left(\hat{\beta}_{1}\right)$, is defined as:

$$
\operatorname{RE}\left(\hat{\beta}_{1}\right)=\frac{\operatorname{Var}\left(\hat{\beta}_{1} \mid \xi_{\text {equal }}\right)}{\operatorname{Var}\left(\hat{\beta}_{1} \mid \xi_{\text {unequal }}\right)} .
$$

Commonly, $\hat{\beta}_{1}$ is the ML estimator, and for this case, an expression for the ARE in Eqn (2) can be derived. Let $\bar{m}^{t}$ and $\bar{m}^{c}$ denote the average cluster size of the $K_{t}$ and $K_{c}$ clusters in the treatment and control arm, respectively. Let $w_{j}^{t}$ and $w_{j}^{c}$ be defined as $w_{j}^{t}=\left(\sigma_{u}^{2}+\sigma_{\delta}^{2} / m_{j}^{t}\right)^{-1}$ and $w_{j}^{c}=\left(\sigma_{v}^{2}+\sigma_{\varepsilon}^{2} / m_{j}^{c}\right)^{-1}$. For equal cluster sizes in the treated arm, we have $m_{j}^{t}=\bar{m}^{t}$ for $j=1, \ldots, K_{t}$, and the weight $w_{j}^{t}$ is denoted as $w_{e}^{t}$. Similarly, for equal cluster sizes in the control arm, we have $m_{j}^{c}=\bar{m}^{c}$ for $j=$ $K_{t}+1, \ldots, K_{t}+K_{c}$, and the weight $w_{j}^{c}$ is denoted as $w_{e}^{c}$. In Appendix A, it is shown that the ARE for the ML estimator $\hat{\beta}_{1}$ then is

$$
R E\left(\hat{\beta}_{1}\right)=\left(\frac{\frac{1}{K_{t} w_{e}^{t}}+\frac{1}{K_{c} w_{e}^{c}}}{\frac{1}{\sum_{j=1}^{K_{t}} w_{j}^{t}}+\frac{1}{\sum_{j=K_{t}+1}^{K_{t}+K_{c}} w_{j}^{c}}}\right)=\left(\begin{array}{c}
\frac{\frac{K_{c} w_{e}^{c}}{K_{t} w_{e}^{t}}+1}{\frac{\frac{K_{c} w_{e}^{c}}{K_{t} w_{e}^{t}}+1}{\sum_{j=1}^{K_{t}} w_{e}^{c}}+\frac{K_{c} w_{e}^{c}}{K_{j}^{t}+K_{c}} w_{j=K_{t}+1}^{c} w_{j}^{c}} \\
\frac{K_{c} w_{e}^{c}}{K_{t} w_{e}^{t}} \times \frac{K_{t} w_{e}^{t}}{\sum_{j=1}^{K_{t}} w_{j}^{t}}+\frac{K_{c} w_{e}^{c}}{\sum_{j=K_{t}+1}^{K_{t}+K_{c}} w_{j}^{c}}
\end{array}\right)
$$

Equation (3) is the weighted harmonic mean of $\sum_{j=1}^{K_{t}} w_{j}^{t} /\left(K_{t} w_{e}^{t}\right)$ and $\sum_{j=K_{t}+1}^{K_{t}+K_{c}} w_{j}^{c} /\left(K_{c} w_{e}^{c}\right)$ with weights $\frac{K_{c} w_{e}^{c}}{K_{t} w_{e}^{t}}$ and 1 , respectively. We define $\psi=\sigma_{y_{t}}^{2} / \sigma_{y_{c}}^{2}$, which is the treatment-to-control ratio of total outcome 
variance. Because $\frac{K_{c} w_{e}^{c}}{K_{t} w_{e}^{t}}=\psi\left(K_{c} / K_{t}\right)\left(\bar{m}^{c} / \bar{m}^{t}\right)\left(\left(\left(\bar{m}^{t}-1\right) \rho_{t}+1\right) /\left(\left(\bar{m}^{c}-1\right) \rho_{c}+1\right)\right)$, the ARE approaches $\sum_{j=1}^{K_{t}} w_{j}^{t} /\left(K_{t} w_{e}^{t}\right)$ if the variance ratio $\psi$, or the allocation ratio $K_{c} / K_{t}$, or the average cluster size $\bar{m}^{c}$ in the control arm, or the intraclass correlation $\rho_{t}$ in the treated arm, increases. On the other hand, if $\psi$, or $K_{c} / K_{t}$ decreases, or if the average cluster size $\bar{m}^{t}$ in the treated arm or the intraclass correlation $\rho_{c}$ in the control arm increases, then the ARE approaches $\sum_{j=K_{t}+1}^{K_{t}+K_{c}} w_{j}^{c} /\left(K_{c} w_{e}^{c}\right)$. It has been shown that $\sum_{j=1}^{K_{t}} w_{j}^{t} /\left(K_{t} w_{e}^{t}\right)$ and $\sum_{j=K_{t}+1}^{K_{t}+K_{c}} w_{j}^{c} /\left(K_{c} w_{e}^{c}\right)$ are the relative efficiencies in cluster randomized trials for estimators of the treatment and control mean, respectively [19]. So the relative efficiency of $\hat{\beta}_{1}$ actually is a weighted harmonic mean of the relative efficiencies for the means of the two arms. Because also $\frac{K_{c} w_{e}^{c}}{K_{t} w_{e}^{t}}=\left(K_{c} / K_{t}\right)\left(\left(\sigma_{u}^{2}+\sigma_{\delta}^{2} / \bar{m}^{t}\right) /\left(\sigma_{v}^{2}+\sigma_{\varepsilon}^{2} / \bar{m}^{c}\right)\right)$, the weight of an arm increases with its outcome variance at either cluster or subject level, and decreases with its sample size at either level, keeping variances and sample sizes in the other arm constant. Van Breukelen et al. [19] have shown that the ARE of varying versus equal cluster sizes within an arm does not depend on the absolute number of clusters (here: $K_{t}$ resp. $K_{c}$ ) and does not exceed 1, but approaches 1 as the intraclass correlation approaches 0 or 1 . As a result, the following properties are true for $\operatorname{RE}\left(\hat{\beta}_{1}\right)$ :

(1) $R E\left(\hat{\beta}_{1}\right)$ does not depend on the cluster numbers in each of the treatment arms, as long as the ratio $K_{t} / K_{c}$ remains the same.

(2) If $\rho_{t} \rightarrow 0$ and $\rho_{c} \rightarrow 0$ or $\rho_{t} \rightarrow 1$ and $\rho_{c} \rightarrow 1$, then $R E\left(\hat{\beta}_{1}\right) \rightarrow 1$.

(3) For $0<\rho_{t}<1$ or $0<\rho_{c}<1$, it holds that $R E\left(\hat{\beta}_{1}\right) \leq 1$ : equal cluster sizes are most efficient.

\section{Taylor approximation of the asymptotic relative efficiency}

To plan sample sizes, it will be useful to have an approximation of the efficiency loss that does not need an exact specification of the cluster size distribution. For such practical purposes, we will present a second-order Taylor approximation of the ARE in Eqn (3), which only depends on the mean and variance of the cluster size distribution. The approximation is based on a stochastic cluster size distribution. Eqn (3) expresses the relative efficiency for a particular realization from such a stochastic cluster size distribution. Second-order Taylor approximations have been derived for the ARE when estimating the mean in the treatment or control arm of a cluster randomized trial [19]. Because the ARE for the treatment effect in a cluster randomized trial with treatment arms that are heterogeneous in terms of the intraclass correlation, outcome variance, average cluster size and cluster numbers is the weighted harmonic mean of the relative efficiencies for the two arm-specific outcome means, a Taylor approximation for Eqn (3) immediately follows from Van Breukelen et al. [19].

Consider $m_{j}^{t}$ for $j=1, \ldots, K_{t}$ and $m_{j}^{c}$ for $j=K_{t}+1, \ldots, K_{t}+K_{c}$ in Eqn (3) as independent realizations of random variables with expectations $\mu_{m}^{t}$ and $\mu_{m}^{c}$, and standard deviations $\sigma_{m}^{t}$ and $\sigma_{m}^{c}$, respectively. Let $C V_{t}=\sigma_{m}^{t} / \mu_{m}^{t}$ and $C V_{c}=\sigma_{m}^{c} / \mu_{m}^{c}$ be the coefficients of variation of cluster sizes for the treatment and control arm, respectively. Furthermore, let $\lambda_{t}=\mu_{m}^{t} /\left(\mu_{m}^{t}+\left(1-\rho_{t}\right) / \rho_{t}\right)$, and let $\lambda_{c}=$ $\mu_{m}^{c} /\left(\mu_{m}^{c}+\left(1-\rho_{c}\right) / \rho_{c}\right)$. The second-order Taylor approximation of the ARE in Eqn (3) can then be derived as the weighted harmonic mean of $1-C V_{t}^{2} \lambda_{t}\left(1-\lambda_{t}\right)$ and $1-C V_{c}^{2} \lambda_{c}\left(1-\lambda_{c}\right)$, the second-order Taylor approximations of the ARE for the treatment and control mean [19], with weights $\psi\left(K_{c} / K_{t}\right)\left(\lambda_{d} / \lambda_{t}\right)\left(\rho_{t} / \rho_{c}\right)$ and 1, respectively:

$$
R E_{T}=\left(K_{c} \lambda_{c} \rho_{t} \psi+K_{t} \lambda_{t} \rho_{c}\right)\left[\left(\frac{K_{c} \lambda_{c} \rho_{t} \psi}{1-C V_{t}^{2} \lambda_{t}\left(1-\lambda_{t}\right)}\right)+\left(\frac{K_{t} \lambda_{t} \rho_{c}}{1-C V_{c}^{2} \lambda_{c}\left(1-\lambda_{c}\right)}\right)\right]^{-1} .
$$

The Taylor approximation in Eqn (4) generalizes the expression derived by Van Breukelen et al. (p. 2594) [19] for the case of trials with homogeneous clustering. As the variance at the 
cluster or subject level in the treatment arm, $\sigma_{u}^{2}$ and $\sigma_{\delta}^{2}$, increases, or its sample size at either level, $K_{t}$ or $\mu_{m}^{t}$, decreases, Eqn (4) approaches $1-C V_{t}^{2} \lambda_{t}\left(1-\lambda_{t}\right)$. Likewise, as the variance at the cluster or subject level in the control arm, $\sigma_{v}^{2}$ and $\sigma_{\varepsilon}^{2}$, increases or its sample size at either level, $K_{c}$ or $\mu_{m}^{c}$, decreases, Eqn (4) approaches $1-C V_{c}^{2} \lambda_{c}\left(1-\lambda_{c}\right)$. Furthermore, when $\rho_{t} \rightarrow 0$ and $\rho_{c} \rightarrow 0$ (and thus $\lambda_{t} \rightarrow 0$ and $\lambda_{c} \rightarrow 0$ ) or $\rho_{t} \rightarrow 1$ and $\rho_{c} \rightarrow 1$ (and thus $\lambda_{t} \rightarrow 1$ and $\lambda_{c} \rightarrow 1$ ), $R E_{T} \rightarrow 1$, and for $0<\rho_{t}$, $\rho_{c}<1, R E_{T} \leq 1$. As $C V_{t}$ and $C V_{c}$ increase, $R E_{T}$ will decrease, and if $\lambda_{t}=\lambda_{c}=1 / 2$, then $1-C V_{t}^{2} \lambda_{t}\left(1-\lambda_{t}\right)$ and $1-C V_{c}^{2} \lambda_{c}\left(1-\lambda_{c}\right)$ each reach their minimum. Therefore, denoting by $C V_{\max }$ the maximum of $C V_{t}$ and $C V_{c}$, the minimum value of Eqn (4) is $\left(1-C V_{\max }^{2} / 4\right)$.

The approximation of the RE as derived in Eqn (4) can be compared with an approximation based on an analysis involving cluster size weighting (cf. $[18,23])$. In this case, the relative efficiency also is a weighted harmonic mean of the relative efficiencies in each of the treatment conditions, with the same weight per arm as aforementioned for Eqn (4). Because the RE for the mean of the treatment and control condition can then be approximated by $1 /\left(1+C V_{t}^{2} \lambda_{t}\right)$ and $1 /\left(1+C V_{c}^{2} \lambda_{c}\right)$, respectively (cf. [23]), the following approximation is obtained based on cluster size weighting:

$$
R E_{c s w}=\left(K_{c} \lambda_{c} \rho_{t} \psi+K_{t} \lambda_{t} \rho_{c}\right)\left[K_{c} \lambda_{c} \rho_{t} \psi\left(1+C V_{t}^{2} \lambda_{t}\right)+K_{t} \lambda_{t} \rho_{c}\left(1+C V_{c}^{2} \lambda_{c}\right)\right]^{-1} .
$$

For homogeneous cluster randomized trials, it has been shown that the approximation based on cluster size weighting gives a rather pessimistic estimate of the efficiency loss in case of (restricted) ML estimation in a mixed effects model, and is less close to the true efficiency loss than the second-order Taylor approximation ([20]). This will also be the case for the efficiency loss as predicted by Eqn (5), which is based on cluster size weighting.

\section{Monte Carlo investigation of the relative efficiency}

We will examine the accuracy of the ARE in Eqn (3) and its Taylor approximation in Eqn (4) through a Monte Carlo simulation study. More specifically, we will calculate the ratio of the simulated variance of the treatment estimator for equal cluster sizes to the simulated variance of the treatment estimator for unequal cluster sizes, and compare that ratio with Eqns (3) and (4). We will allow the intraclass correlations, the variances within treatment conditions, the mean cluster sizes and the number of clusters to differ between the treatment arms. Randomization may be assumed to imply similar shapes of the cluster size distributions in the two treatment arms, but the coefficient of variation may be different because the planned average cluster size may differ between arms, that is, $\bar{m}^{t} \neq \bar{m}^{c}$.

\subsection{Design of the simulation study}

In the simulation study, the intercept $\beta_{0}$ and the effect parameter $\beta_{1}$ in Eqn (1) were fixed at 50 and 5, respectively. This could be done without loss of generality, because the asymptotic variance of the effect estimator is independent of the fixed regression weights (see Eqn (A2) in appendix A). The following factors were varied systematically in the simulation study, as they enter into Eqns (3) and (4): (i) the frequency distribution of the cluster sizes; (ii) the size of the intraclass correlations $\rho_{t}$ and $\rho_{c}$; (iii) the variance ratio $\psi$; (iv) the ratio of cluster numbers $K_{t} / K_{c}$ and (v) the average cluster sizes

\begin{tabular}{|l|l|}
\hline \multicolumn{2}{|l|}{ Table I. Overview of the conditions of the Monte Carlo simulation } \\
\hline Factor & Levels \\
\hline Cluster size distribution $^{1}$ & Unimodal, uniform, bimodal, positively skewed, \\
& negatively skewed distribution \\
Intraclass correlations & $\rho_{t}, \rho_{c}=0.01$ up to 0.29 \\
Ratio of variances (treatment vs control arm) & $\psi=0.25,1,4$ \\
Average cluster size & $\left(\bar{m}^{t}, \bar{m}^{c}\right)=(4,4),(10,4)$ or $(10,10)$ \\
Ratio of cluster numbers (treatment vs control arm) & $K_{t} / K_{c}=0.25(6 / 24), K_{t} / K_{c}=1(12 / 12) K_{t} / K_{c}=4(24 / 6)$ \\
Estimation method & $\mathrm{ML}, \mathrm{REML}$ \\
\hline
\end{tabular}

${ }^{1}$ More details on the different cluster size distributions are given in Table $2 \mathrm{a}$ and $\mathrm{b}$.

${ }^{2} \mathrm{~A}$ larger average cluster size of 10 enables examining the effect of a larger range of cluster sizes and of average cluster sizes differing between the treatment arms.

ML, maximum likelihood; REML, restricted maximum likelihood. 
$\bar{m}^{t}$ and $\bar{m}^{c}$. In addition, the estimation method was varied. Table 1 displays the choices made for these factors, the motivation will be given below.

\subsubsection{Distribution of cluster sizes}

Five different cluster size distributions were studied: (i) a unimodal; (ii) a uniform; (iii) a bimodal; (iv) a positively skewed and (v) a negatively skewed distribution. Details of the cluster size distributions can be found in Table $2 \mathrm{a}$ and $\mathrm{b}$.

\begin{tabular}{|c|c|c|c|c|c|c|c|}
\hline \multirow[t]{2}{*}{ Distribution } & \multicolumn{3}{|c|}{ Cluster sizes } & \multicolumn{3}{|c|}{ Cluster frequencies } & \multirow{2}{*}{$\begin{array}{l}\text { Coefficient of variation } \\
(C V) \text { of cluster sizes }\end{array}$} \\
\hline & $g_{a}$ & $g_{b}$ & $g_{c}$ & $f_{a}$ & $f_{b}$ & $f_{c}$ & \\
\hline \multicolumn{8}{|l|}{ (a) } \\
\hline \multicolumn{8}{|l|}{ Unimodal } \\
\hline$K_{t}=24$ & 2 & 4 & 6 & 6 & 12 & 6 & 0.36 \\
\hline$K_{t}=12$ & 2 & 4 & 6 & 2 & 8 & 2 & 0.30 \\
\hline$K_{t}=6$ & 2 & 4 & 6 & 1 & 4 & 1 & 0.32 \\
\hline \multicolumn{8}{|l|}{ Uniform } \\
\hline$K_{t}=24$ & 2 & 4 & 6 & 8 & 8 & 8 & 0.42 \\
\hline$K_{t}=12$ & 2 & 4 & 6 & 4 & 4 & 4 & 0.43 \\
\hline$K_{t}=6$ & 2 & 4 & 6 & 2 & 2 & 2 & 0.45 \\
\hline \multicolumn{8}{|l|}{ Bimodal } \\
\hline$K_{t}=24$ & 2 & 4 & 6 & 11 & 2 & 11 & 0.49 \\
\hline$K_{t}=12$ & 2 & 4 & 6 & 5 & 2 & 5 & 0.48 \\
\hline$K_{t}=6$ & 2 & 4 & 6 & 3 & 0 & 3 & 0.55 \\
\hline \multicolumn{8}{|c|}{ Positively skewed } \\
\hline$K_{t}=24$ & 3 & 4 & 7 & 12 & 8 & 4 & 0.36 \\
\hline$K_{t}=12$ & 3 & 4 & 7 & 6 & 4 & 2 & 0.37 \\
\hline$K_{t}=6$ & 3 & 4 & 7 & 3 & 2 & 1 & 0.39 \\
\hline \multicolumn{8}{|c|}{ Negatively skewed } \\
\hline$K_{t}=24$ & 1 & 4 & 5 & 4 & 8 & 12 & 0.36 \\
\hline$K_{t}=12$ & 1 & 4 & 5 & 2 & 4 & 6 & 0.37 \\
\hline$K_{t}=6$ & 1 & 4 & 5 & 1 & 2 & 3 & 0.39 \\
\hline \multicolumn{8}{|l|}{ (b) } \\
\hline \multicolumn{8}{|l|}{ Unimodal } \\
\hline$K_{t}=24$ & 2 & 10 & 18 & 6 & 12 & 6 & 0.58 \\
\hline$K_{t}=12$ & 2 & 10 & 18 & 2 & 8 & 2 & 0.48 \\
\hline$K_{t}=6$ & 2 & 10 & 18 & 1 & 4 & 1 & 0.51 \\
\hline \multicolumn{8}{|l|}{ Uniform } \\
\hline$K_{t}=24$ & 2 & 10 & 18 & 8 & 8 & 8 & 0.67 \\
\hline$K_{t}=12$ & 2 & 10 & 18 & 4 & 4 & 4 & 0.68 \\
\hline$K_{t}=6$ & 2 & 10 & 18 & 2 & 2 & 2 & 0.72 \\
\hline \multicolumn{8}{|l|}{ Bimodal } \\
\hline$K_{t}=24$ & 2 & 10 & 18 & 11 & 2 & 11 & 0.78 \\
\hline$K_{t}=12$ & 2 & 10 & 18 & 5 & 2 & 5 & 0.76 \\
\hline$K_{t}=6$ & 2 & 10 & 18 & 3 & 0 & 3 & 0.88 \\
\hline \multicolumn{8}{|c|}{ Positively skewed } \\
\hline$K_{t}=24$ & 6 & 10 & 22 & 12 & 8 & 4 & 0.58 \\
\hline$K_{t}=12$ & 6 & 10 & 22 & 6 & 4 & 2 & 0.59 \\
\hline$K_{t}=6$ & 6 & 10 & 22 & 3 & 2 & 1 & 0.62 \\
\hline \multicolumn{8}{|c|}{ Negatively skewed } \\
\hline$K_{t}=24$ & 1 & 10 & 17 & 7 & 8 & 9 & 0.66 \\
\hline$K_{t}=12$ & 1 & 8 & 17 & 3 & 4 & 5 & 0.68 \\
\hline$K_{t}=6$ & 1 & 4 & 17 & 1 & 2 & 3 & 0.78 \\
\hline
\end{tabular}

${ }^{1} f_{a}$, number of clusters of size $g_{a}$ (small); $f_{b}$, number of clusters of size $g_{b}$ (medium); $f_{c}$, number of clusters of size $g_{c}$ (large).

${ }^{2}$ The range of the cluster sizes $=g_{c}-g_{a}=4$.

${ }^{3}$ The range of the cluster sizes $=g_{c}-g_{a}=16$.

${ }^{4}$ Coefficient of variation is the standard deviation of cluster sizes divided by the mean cluster size. 


\section{Statistics}

\subsubsection{Intraclass correlations}

The intraclass correlations $\rho_{t}$ and $\rho_{c}$ range from 0.01 to 0.30 , because this represents the range commonly encountered in trials comparing group administered interventions [2,4,5], trials where clustering effects are due to therapists $[2,24]$ and in cluster randomized trials $[25,26]$.

\subsubsection{Ratio of outcome variances}

Because there is not much empirical evidence on $\psi$, with studies indicating that it varies between 0.5 and 2 [4], we will examine a somewhat broader range: $\psi$ runs from 0.25 to 4 .

\subsubsection{Ratio of the number of clusters}

The $R E\left(\hat{\beta}_{1}\right)$ does not depend on the total number of clusters but does depend on the ratio $K_{t} / K_{c}$. In most studies on group therapy (e.g. [4,27-29]), but also in cluster randomized trials (e.g. [26,30]), the cluster numbers are very similar for both treatment arms. Because it has been shown that unequal cluster numbers may be optimal under heterogeneity of outcome variance [13], in our simulations, next to $K_{t} / K_{c}=1$, we will also consider $K_{t} / K_{c}=0.25$ and $K_{t} / K_{c}=4 . K_{t}$ and $K_{c}$ were deliberately chosen to be small in order to check the accuracy of the asymptotic expression in Eqn (3), but also large enough to provide sufficient power in a reasonable number of cases. Three sets of values were considered: (i) $K_{t}=K_{c}=12$; (ii) $K_{t}=24$ and $K_{c}=6$ and (iii) $K_{t}=6$ and $K_{c}=24$.

\subsubsection{Average cluster sizes}

An average cluster size of four was chosen, with a range of four, because this is about the smallest average cluster size encountered (especially in group therapy see, for instance, [5,29,31]). An average cluster size of 10 was also examined, because this allows for a larger range of cluster sizes. More specifically, a rather extreme range of 16 was chosen, which, for symmetrical distributions, implies that the smallest $m=2$ and the largest $m=18$. The cluster sizes were deliberately chosen small in order to check the accuracy of the asymptotic expression in Eqn (3). Simulations were performed with (i) an average cluster size $\bar{m}^{t}=\bar{m}^{c}=4$ for both the treatment and control arm, (ii) an average cluster size $\bar{m}^{t}=10$ for the treatment and an average cluster size $\bar{m}^{c}=4$ for the control arm, and (iii) an average cluster size $\bar{m}^{t}=\bar{m}^{c}=10$ for both arms. In many studies (e.g. [4,29,30]), the ratio of average cluster sizes $\bar{m}^{t} / \bar{m}^{c}$ is close to 1 , but we also consider the case with $\bar{m}^{t} / \bar{m}^{c}=10 / 4=2.5$. Note that Eqn (3) shows that the ARE does not depend on the treatment effect itself, so that the ratio $\bar{m}^{t} / \bar{m}^{c}=4 / 10=0.4$ is redundant when all combinations of levels of all other design factors have been made. This is the case, except for the coefficient of cluster size variation which is large for large cluster sizes and small for small cluster sizes; see Table $2 \mathrm{a}$ and $\mathrm{b}$. To check the validity of our conclusions, we therefore also performed 35 additional simulations with coefficients of cluster size variation for small cluster sizes that are comparable with the coefficients of cluster size variation for large cluster sizes, and vice versa.

\subsection{Simulation procedure and estimation methods}

For each of the simulation conditions 10000 data sets were generated, each representing the data for 24 or 30 clusters consisting, on the average, of 4 or 10 persons. The simulations as well as model estimation were performed in MLWIN, version 2.0 [32]. For the analysis model as specified in Eqn (1), ML and REML estimates were obtained through the 'Iterative Generalized Least Squares' and 'Restricted Iterative Generalized Least Squares' algorithm, respectively, with negative estimates of the variance components being truncated to 0 . Nontruncation of negative variance estimates in many cases led to nonconvergence, and therefore was not considered further. The convergence criterion was set to 0.001, that is, the estimation algorithm stopped when the relative change for all parameter estimates was less than 0.001. To speed up the simulations, the number of iterations was maximized at 200, resulting in less than $1 \%$ nonconverged runs, and for the majority of simulation scenarios even less than $0.5 \%$ nonconverged runs. Based on the treatment effect estimates, the sampling variance of $\hat{\beta}_{1}$ was calculated for equal and for varying cluster sizes, allowing the RE in Eqn(2) to be calculated as the golden standard against which the approximations in Eqns (3), (4) and (5) could be compared. Detailed codes for the programs MLWIN, SPSS and R to run the analysis model in Eqn (1) are provided as supporting information. 

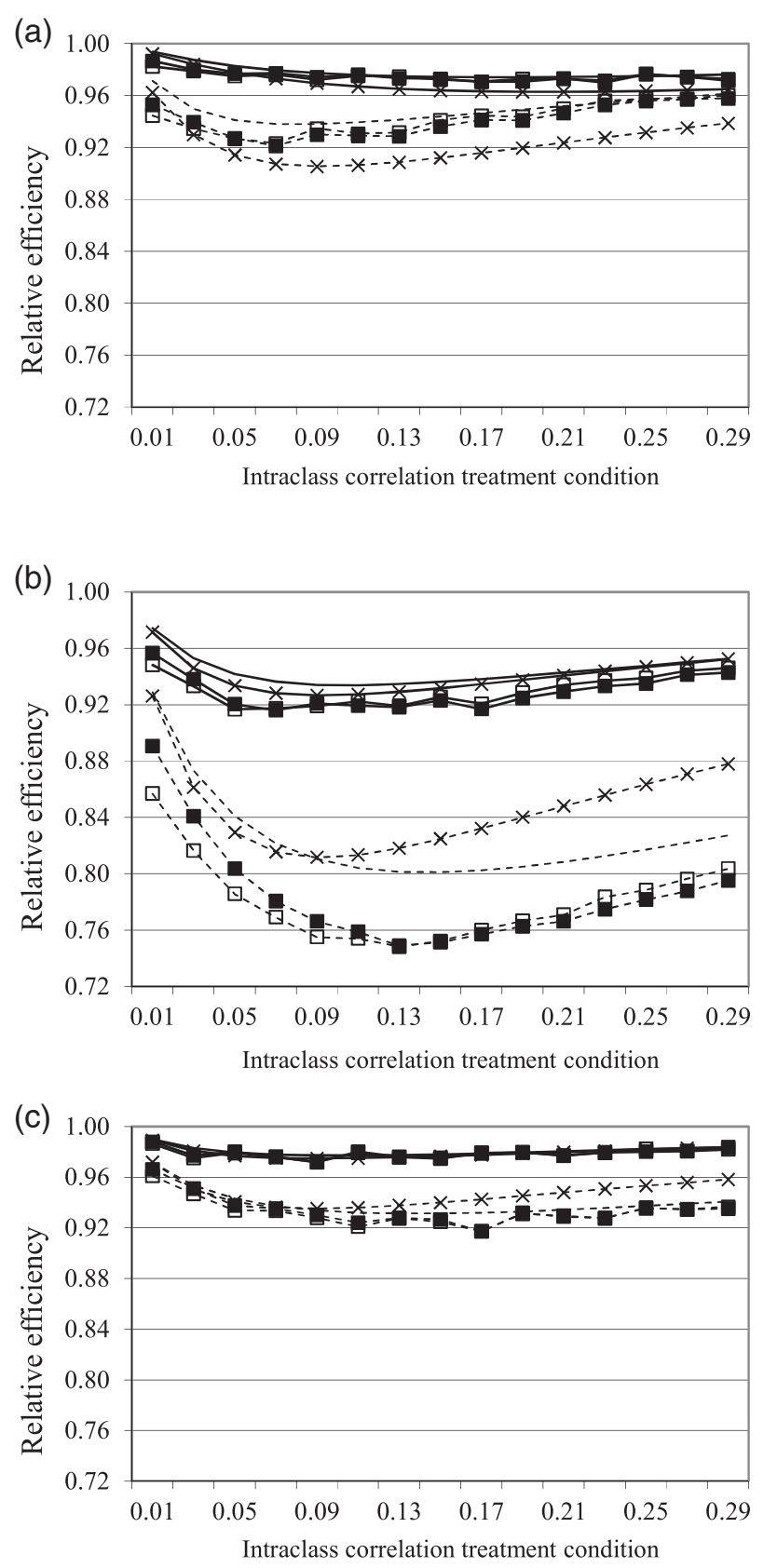
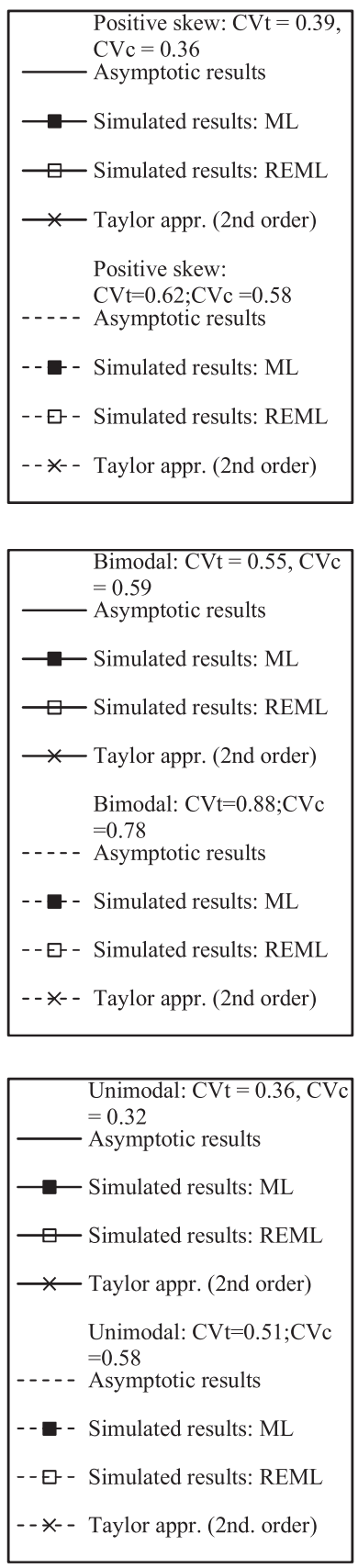

Figure 1. Relative efficiency of the treatment effect estimator for a positively skewed (a), bimodal (b) and unimodal distribution of cluster sizes (c) as a function of the intraclass correlation in the treatment condition, $\rho_{t}$, with $\psi=4, \rho_{c}=0.15, K_{t} / K_{c}=1 / 4$. The top curves in (a) and (b) are for $\bar{m}^{t}=\bar{m}^{c}=4$, all other curves are for $\bar{m}^{t}=\bar{m}^{c}=10$. The asymptotic relative efficiency of the ML estimator and its Taylor approximation are also displayed. CV: coefficient of cluster size variation.

\subsection{Results for homogeneous average cluster sizes}

For $\psi=4, K_{t} / K_{c}=1 / 4$ and $\rho_{c}=0.15$ Figure 1 illustrates the results of distributions that give extreme results in terms of the RE: the unimodal and positively skewed distribution on the one hand and the bimodal distribution on the other hand. The top curves in Figure 1a and b are for $\bar{m}^{t}=\bar{m}^{c}=4$, the bottom curves are for $\bar{m}^{t}=\bar{m}^{c}=10$. In Figure 1c, all curves are for $\bar{m}^{t}=\bar{m}^{c}=10$. Although changing the between-arm ratio of outcome variances $\psi$ or the between-arm ratio of cluster numbers $K_{t} / K_{c}$ yields similar results, the present values are chosen for later comparison with results for heterogeneous average cluster sizes (Section 5.4). Further, we chose $\rho_{c}=0.15$ as some intermediate value of the interval [0.01, 0.30]. The effects of changing $\rho_{c}$ are comparable with the effects of changing $\rho_{t}$ as visible in Figure 1 . 


\section{Statistics}

In line with the ARE, Figure 1 shows that the simulated RE approaches 1 when $\rho_{t} \rightarrow 0$ and never exceeds 1 , implying that varying cluster sizes are less efficient than constant cluster sizes. Also, the simulated RE hardly differs between REML and ML. As illustrated by the top curves in Figure 1a, for $\bar{m}^{t}=\bar{m}^{c}=4$, the asymptotic and simulated RE are rather close in that the simulated RE is overestimated by less than $2 \%$. The exceptions are the negatively skewed distribution and bimodal distribution where the overestimation can become 3\% and 4\%, respectively. This is illustrated by the upper curves in Figure 1b. Similar conclusions can be drawn for the accuracy of the second-order Taylor approximation in Eqn (4).

As illustrated by the lower curves of Figure 1a-c, for $\bar{m}^{t}=\bar{m}^{c}=10$, the ARE is less close to the simulated RE than for $\bar{m}^{t}=\bar{m}^{c}=4$, overestimating the RE up to $9 \%$. This also holds for the second-order Taylor approximation (Eqn (4)), overestimating the simulated RE in many cases by 4\% (but up to $10 \%$ ). The larger approximation errors of the ARE and its Taylor approximation for $\bar{m}^{t}=\bar{m}^{c}=10$, when compared with $\bar{m}^{t}=\bar{m}^{c}=4$, can be attributed to the larger coefficients of cluster size variation. Figure 1c illustrates this for a unimodal distribution and $\bar{m}^{t}=\bar{m}^{c}=10$, where the upper curves are for small coefficients of cluster size variation and the lower curves are for large coefficients of cluster size variation. For the larger coefficients of cluster size variation, the approximation errors are larger.

\subsection{Results for heterogeneous average cluster sizes}

For the case $\bar{m}^{t}=10$ and $\bar{m}^{c}=4, \psi=4, K_{t} / K_{c}=1 / 4$ and $\rho_{c}=0.15$, Figure 2 shows results for a positively skewed, bimodal and unimodal distribution. To examine the largest efficiency loss, results are shown for designs where the arm with the largest $C V$ (i.e. $\bar{m}=10$ ) also has the largest outcome variance and the smallest number of clusters, such that this arm gets the largest weight in the relative efficiency according to Eqn (3). Similar to what was observed in Figure 1, the differences in RE between ML and REML were small for the present scenario and Figure 2 therefore only shows the REML results. In line with the ARE, the simulated RE in Figure 2 does not exceed 1 (but approaches 1 when $\rho_{t} \rightarrow 0$ ). It can also be seen that the minimum REs in Figure $2 \mathrm{a}$ and $\mathrm{b}$ are clearly lower than the minimum REs for the solid lines in Figure 1a and b, but higher than the minimum REs for the dashed lines in Figure 1a and $b$. This is in line with Eqn (3), which defines the ARE as the weighted harmonic mean of the relative efficiencies of each treatment arm, with one arm in Figure 2a and b having a $C V$ identical to the $C V$ of the solid lines and the other arm having a $C V$ identical to the $C V$ of the dashed lines in Figure 1a and b, respectively.

Figure 2 also illustrates that, compared with designs with $\bar{m}^{t}=\bar{m}^{c}=4$, the asymptotic and the simulated RE are less close. The difference between the asymptotic and the simulated RE is less than $4 \%$, except for a negatively skewed and bimodal distribution where the RE may be overestimated up to $5 \%$ and $9 \%$, respectively. The approximation errors of the second-order Taylor approximation (Eqn (4)) can become rather large (up to $10 \%$ ).

Finally, Figure 2 illustrates the improvements of the Taylor approximation derived in this study (Eqn (4)), as compared with the approximation based on cluster size weighting (Eqn (5)). Particularly for larger intraclass correlations, the approximation in Eqn (5) overestimates the efficiency loss to a large extent (cf. [20]).

Table 3 summarizes the minimum values for the simulated RE as well as the maximum overestimations of the simulated RE by the ARE and its Taylor approximation. The minima and maxima are examined across all intraclass correlations $\rho_{t}$ and $\rho_{c}$, ratios $K_{t} / K_{c}$ and $\psi$. The results on overestimation can be used in sample size planning to correct the ARE or its Taylor approximation. If there is not enough information to calculate the ARE or its Taylor approximation, the minimum of the Taylor approximation can be used, that is, $\left(1-C V_{\max }^{2} / 4\right)$, only requiring the maximum of $C V_{t}$ and $C V_{c}$. Table 3 therefore also displays how much $\left(1-C V_{\max }^{2} / 4\right)$ maximally overestimates the minimum of the simulated RE.

As illustrated by Table 3, the minimum of the simulated RE depends on the type of cluster size distribution and the coefficients of cluster size variation of the treatments arms. The larger the coefficient of cluster size variation, the smaller the minimum simulated RE. More detailed analyses (not shown) furthermore show, when keeping the coefficient of cluster size variation, $K_{t} / K_{c}, \psi$, and $\bar{m}^{t}$ and $\bar{m}^{c}$ constant, that the minimum is smallest for a negatively skewed distribution and largest for a positively skewed distribution, with the symmetrical distributions being in between. There are no substantial effects of $K_{t} / K_{c}, \psi$, and $\bar{m}^{t}$ and $\bar{m}^{c}$ on the minimum simulated RE.

Table 3 furthermore illustrates that the approximation errors increase with increasing coefficients of cluster size variation of the treatment arms. From more detailed analyses (not shown) it appears that, when keeping the coefficient of cluster size variation, $K_{t} / K_{c}, \psi$, and $\bar{m}^{t}$ and $\bar{m}^{c}$ constant, that the maximum overestimations 
(a)

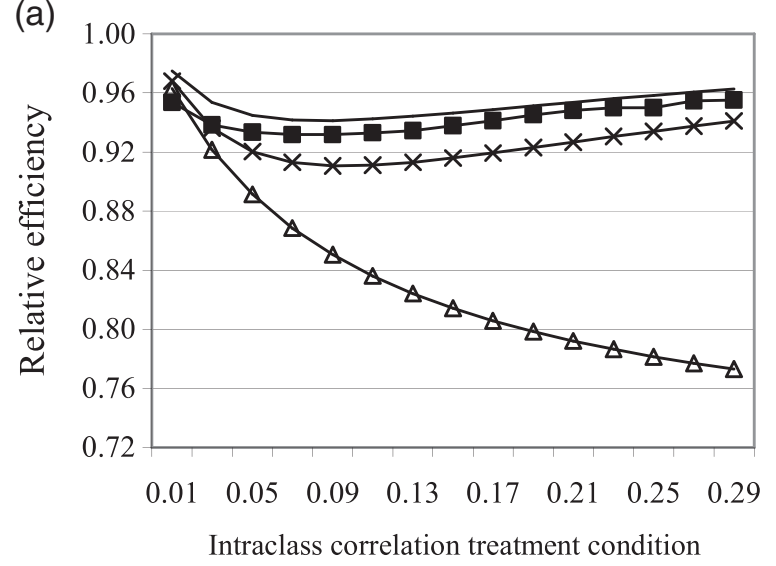

(b)

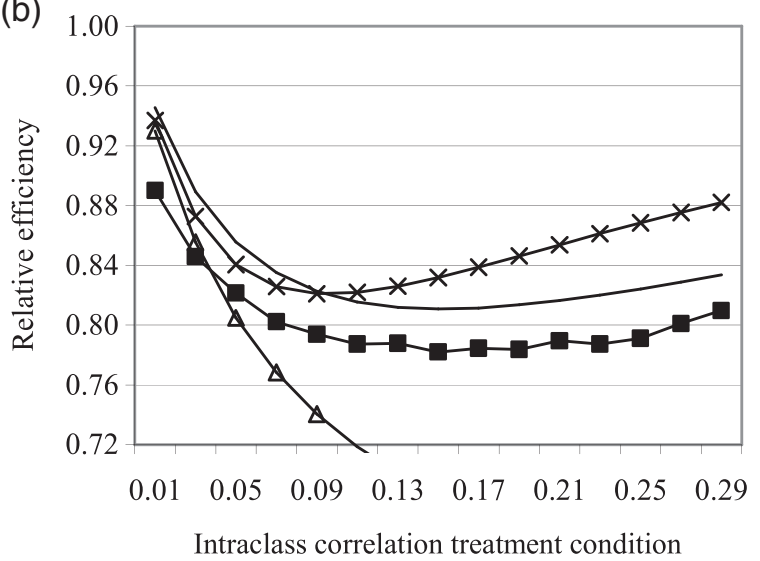

(c)

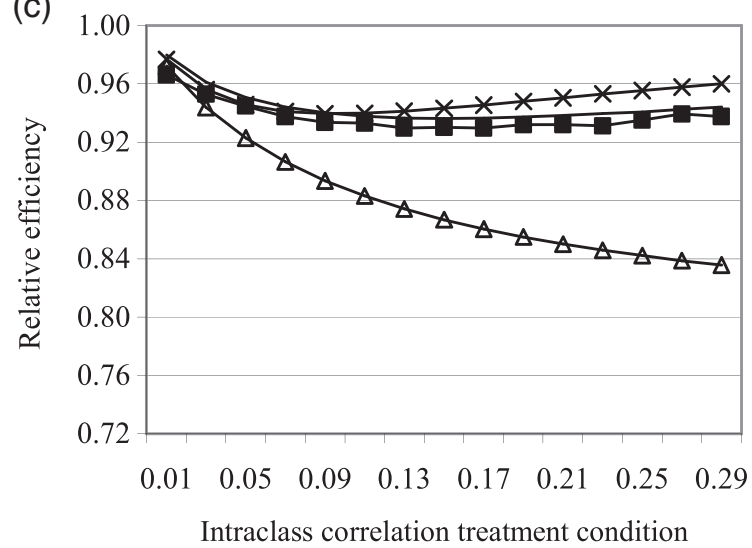


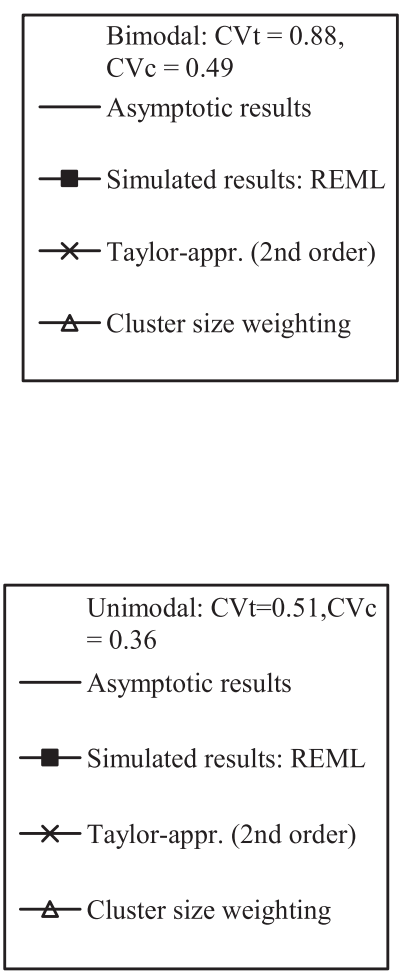

Figure 2. Relative efficiency of the treatment effect estimator for a positively skewed (a), bimodal (b) and unimodal distribution of cluster sizes (c), as a function of the intraclass correlation in the treatment condition, $\rho_{t}$, with $\psi=4, \rho_{c}=0.15$ and $K_{t} / K_{c}=1 / 4$, and $\bar{m}^{t}=10$ in the treatment arm and $\bar{m}^{c}=4$ in the control arm. The asymptotic relative efficiency of the maximum likelihood (ML) estimator, its Taylor approximation, and the approximation based on cluster size weighting are also displayed. CV: coefficient of cluster size variation.

of the RE by the Taylor approximation and of the minimum simulated RE by $\left(1-C V_{\max }^{2} / 4\right)$ also depend on the type of cluster size distribution. Compared with the symmetric cluster size distributions, the overestimation is smaller for a positively skewed distribution but larger for a negatively skewed distribution. Figure 3 illustrates this for a uniform and negatively skewed distribution of cluster sizes, both with $C V_{t}=C V_{c}=0.68$. The approximation error of the Taylor approximation is clearly larger for the negatively skewed distribution.

\subsection{Guidelines for estimating the efficiency loss}

The results from Table 3 can be used in estimating the efficiency loss. If a researcher has no specific idea on the type of cluster size distribution, the Taylor approximation could be used, that is, Eqn (4), 
Table III. Minimum simulated relative efficiencies (minRE), maximum overestimation of simulated RE by the asymptotic RE (ARE) and Taylor approximation (TRE) and maximum overestimation of minRE by $\left(1-C V_{\max }^{2} / 4\right)$, across all intraclass correlations $\rho_{t}$ and $\rho_{c}$, ratios $K_{t} / K_{c}$ and $\psi$, for different sets of average cluster sizes ( $C V_{\max }$ : largest coefficient of cluster size variation for the two treatment arms).

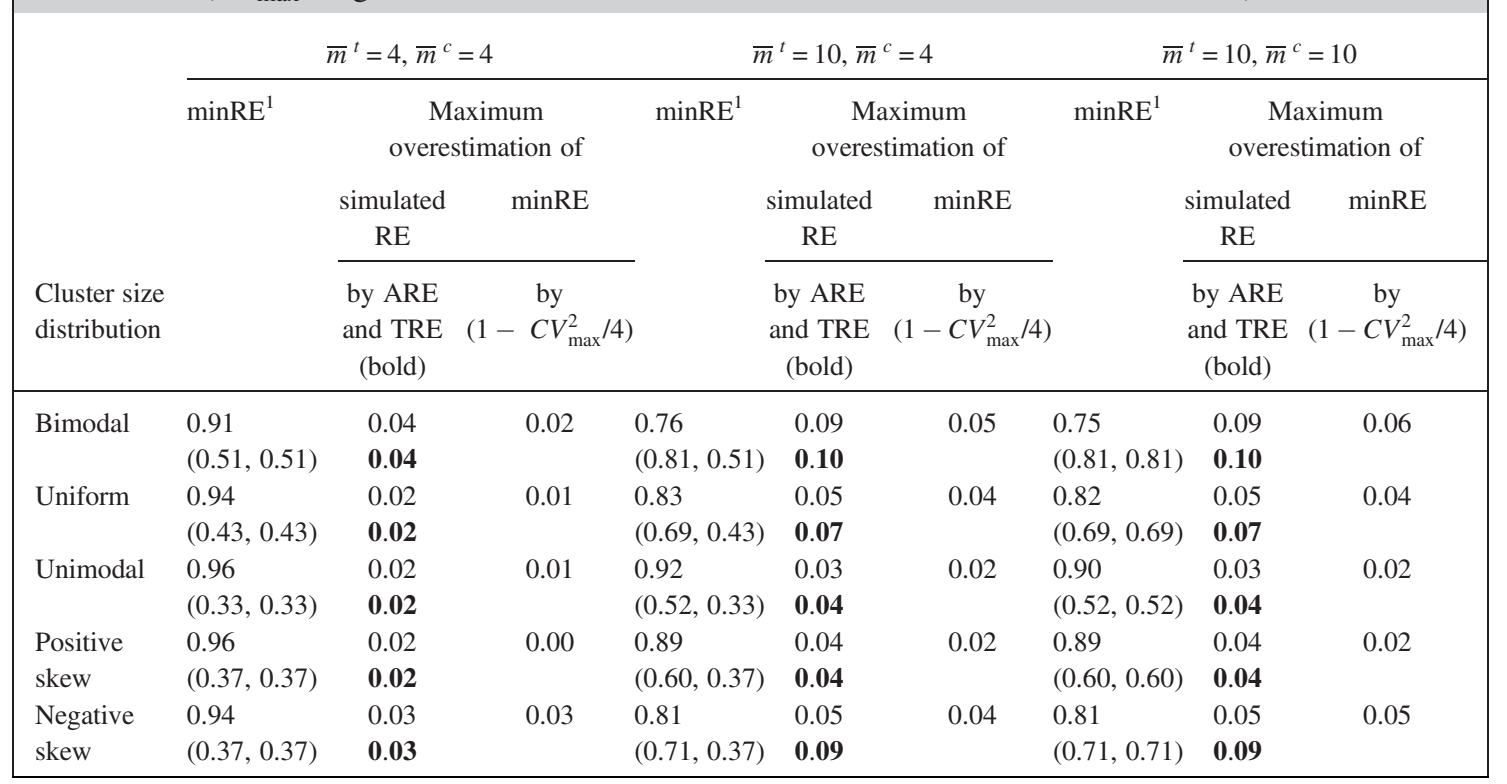

${ }^{1}$ Within brackets the average coefficients of cluster size variation for the treatment and control arm are given. $\mathrm{CV}$ : coefficient of cluster size variation.
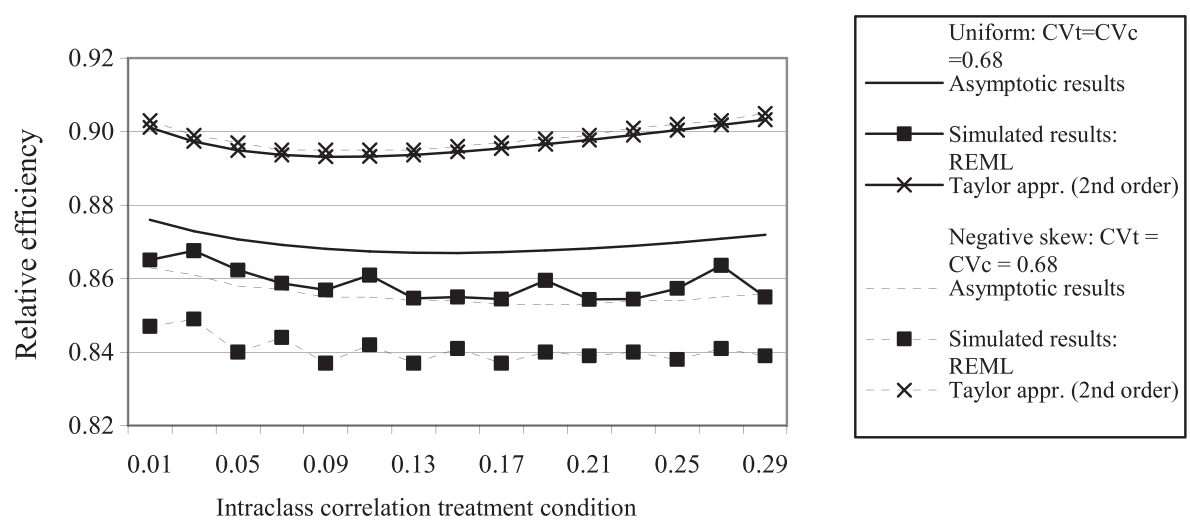

Figure 3. Relative efficiency of the treatment effect estimator for a uniform and negatively skewed distribution of cluster sizes as a function of the intraclass correlation in the treatment condition, $\rho_{t}$, with $\psi=4$, $\rho_{c}=0.15, K_{t} / K_{c}=1$ and $\bar{m}^{t}=\bar{m}^{c}=10$. The asymptotic relative efficiency of the maximum likelihood (ML) estimator and its Taylor approximation are also displayed. CV: coefficient of cluster size variation.

and, if the researcher does not have an idea of relevant model parameters $\left(\rho_{t}, \rho_{c}\right.$ and $\left.\psi\right)$, its lower-bound, $\left(1-C V_{\max }^{2} / 4\right)$ could be used. A pessimistic scenario could then be chosen, by assuming large coefficients of cluster size variation for each of the treatment arms. Next, the resulting RE has to be corrected for possible overestimation according to the results in Table 3. A negatively skewed distribution should be chosen, as this choice leads to a safe correction for overestimating the RE.

If the researcher does have a specific idea of the cluster size distribution as well as of relevant model parameters, then the ARE in Eqn (3) can be calculated. Choosing in Table 3, a large coefficient of cluster size variation for the cluster size distribution considered plausible would then again lead to a safe correction for overestimating the RE. Of course, if a researcher has a detailed idea of both model parameters and the cluster size distribution, also a (rather time intensive) simulation could be performed to estimate the efficiency loss, similar to what was performed in the Monte Carlo simulations. The code for performing such an analysis in MLWIN [32] is, upon request, available from the first author. 


\section{Application in planning a trial}

We illustrate how the results of the present study can be used in planning a trial in which there is heterogeneous clustering in the treatment arms. Appendix B shows that there is only one practical way of restoring the efficiency loss due to varying cluster sizes when estimating the treatment effect. If RE is the trial's relative efficiency of varying versus constant cluster sizes, the efficiency loss can be regained by multiplying the number of clusters in both the treatment and control arm by 1/RE. In optimal designs, the aim is to minimize the costs of a study for a given level of precision and power. When aiming for such a design, it furthermore is shown in Appendix B that multiplying the number of clusters in both the treatment and control arm by $1 / \mathrm{RE}$ does not exactly minimize the costs of a study but is very close to minimizing these costs, in that, in most cases, the smallest possible budget is exceeded by less than $1 \%$.

We apply these results to a randomized trial by Baldwin et al. [4]. High school and university students expressing body image concerns either received a group-administered dissonance intervention or a groupadministered healthy weight management program. A central outcome was the score on the Revised Ideal Body Stereotype Scale, a self-report measure of internalization of the thin beauty ideal. The parameter estimates from Baldwin et al. [4] (i.e. $\hat{\rho}_{t}=0.04, \hat{\rho}_{c}=0.25$ and $\hat{\psi}=0.78$ ) could be used to specify plausible ranges for these parameters: $\rho_{t} \in[0.01,0.10], \rho_{c} \in[0.20,0.30]$ and $\psi \in[0.4,2.5]$. Based on these ranges, a maximin design can be calculated, that is, a design which guarantees a power level within the specified parameter ranges at the lowest costs or with the lowest number of subjects [13]. Assuming Baldwin et al.'s [4] average cluster sizes $\left(\bar{m}^{t}=\bar{m}^{c}=6\right)$ and aiming for $80 \%$ power to detect a medium sized effect, that is, $\beta_{1} / \sqrt{0.5\left(\sigma_{y_{t}}^{2}+\sigma_{y_{c}}^{2}\right)}=0.5$ (cf. Cohen [33]), in a two-tailed test with a $5 \%$ type I error rate, one can calculate, by employing a small menu-driven program CLUSCALCRT in R [13], that $K_{t}=18$ groups for the treatment arm and $K_{c}=29$ groups for the control arm minimize the total sample size.

The present's study simulations show that varying cluster sizes leads to efficiency loss. The cluster size distribution being unknown, one may start from a pessimistic scenario, assuming rather large values for $C V_{t}=C V_{c}=0.70$. A lower-bound for the RE according to the second-order Taylor approximation would be $1-C V_{\max }^{2} / 4=0.88$. Because this RE may underestimate the efficiency loss, a conservative, but safe correction would be lowering the RE by the maximum approximation error found in the present study, occurring for a negatively skewed distribution. For an average $C V$ of 0.71 in both arms, this safe correction is $5 \%$ (see column $\bar{m}^{t}=\bar{m}^{c}=10$ in Table 3), resulting in $R E=0.88-0.05=0.83$. This efficiency loss can then be restored in a (nearly) cost-optimal way by multiplying the number of groups in both arms with $1 / R E=1 / 0.83=1.14$, yielding $K_{t}=21$ and $K_{c}=34$ groups, respectively.

Another approach to repairing the efficiency loss is to employ a simple numerical search, to find the lowest value of the Taylor approximation in Eqn (4) for the specified plausible ranges of the parameters also used in deriving the maximin design, that is, $\rho_{t} \in[0.01,0.10], \rho_{c} \in[0.20,0.30]$, and $\psi \in[0.4,2.5]$. The lowest value turns out to be $R E=0.88$. The simulations show that the RE is most overestimated for a negatively skewed distribution. In case the average $C V$ in both arms is 0.71 , the maximum overestimation, across all intraclass correlations, variance and cluster number ratios, is $9 \%$ (see column $\bar{m}^{t}=\bar{m}^{c}=10$ in Table 3). However, because 0.88 is also the minimum according to $1-C V_{\max }^{2} / 4$, one can infer that we only have to correct this $\mathrm{RE}$ with $5 \%$, resulting in the pessimistic estimate $R E=0.88-0.05=0.83$, yielding a correction factor that is identical to the first approach. Both methods thus lead to restoring the efficiency loss by multiplying the number of groups in both arms with $1 / R E=1 / 0.83=1.14$, yielding $K_{t}=21$ and $K_{c}=34$ groups, respectively.

\section{Conclusions and discussion}

Clustering may occur in cluster randomized trials, where organizational units (e.g. general practices) are assigned to treatments, in trials comparing group administered treatments (e.g. groupwise therapy), or in trials comparing individually administered treatments where each care provider (e.g. a therapist) is assigned a group of persons following one of the treatments. The cluster sizes and numbers of clusters may differ across arms, and analyzing data for such designs by a linear mixed model may require allowance for between-arm differences in the intraclass correlations and outcome variances. For this general case, an expression of the ARE of varying versus constant cluster sizes was derived. Starting from this expression, it was shown that the efficiency loss due to varying cluster sizes can be restored almost costefficiently by increasing the numbers of clusters in both arms by (1/RE) -1 . 
To study the accuracy of the ARE when calculating the efficiency loss for realistic sample sizes, an extensive Monte Carlo simulation was performed. For coefficients of cluster size variation $(\mathrm{CV})$ up to 0.60 in both arms, the ARE was rather close to the simulated RE: lowering the ARE by $4 \%$ would then be a safe correction. For a $C V$ up to 0.80 in one of the arms, the overestimation of the ARE could become larger, up to $9 \%$ (see Table 3 for details). For all designs studied, the simulated RE did not differ much between ML and REML.

To facilitate taking care of the efficiency loss in planning a trial, a second-order Taylor approximation of the ARE was derived. This approximation rather adequately described the simulated RE, the overestimation being less than $4 \%$ if the $C V$ in both arms did not exceed 0.50 , or, for a positively skewed distribution if the $C V$ in both arms did not exceed 0.60 . The Taylor approximation was clearly less accurate with larger $C V \mathrm{~s}$.

If there is no information on the intraclass correlations or the variance ratio, the second-order Taylor approximation provides a simple formula for the minimum RE, $\left(1-C V_{\max }^{2} / 4\right)$, where $C V_{\max }$ is the largest $C V$ of the two arms. This expression can underestimate the efficiency loss, but lowering $\left(1-C V_{\max }^{2} / 4\right)$ by $1 \%$ up to $6 \%$, depending on the $C V$ and type of cluster size distribution (see Table 3 for details), would be sufficient to correct for the efficiency loss in sample size calculation.

For some care providers, such as nurse practitioners or general practitioners, $K_{t} / K_{c}$ may clearly deviate from 1 [34,35], even close to 4 [2]. The range of ratios presently considered also encompasses these between-arm ratios of cluster numbers. However, for these care providers, the intervention often involves a single or a few meetings, of limited duration, implying that the average number of patients per care provider may be much larger than considered in the present simulation study (e.g. Roberts et al. [2]; Roth et al. [35]). Larger cluster sizes are also characteristic for community based trials in which, for instance, villages or neighbourhoods are assigned to different treatments [17]. Designs with large cluster sizes may show larger $C V$ s and larger between-arm heterogeneity in terms of the average cluster sizes. Additional simulations with larger average cluster sizes (between 10 and 120), indicate that, provided the $C V$ does not exceed the values in Table $2 \mathrm{a}$ and $\mathrm{b}$, the results are similar to Table 3 , also for more extreme cluster size ratios of $\bar{m}^{t} / \bar{m}^{c}=5$ and $\bar{m}^{t} / \bar{m}^{c}=10$.

The correction factors for the number of clusters for varying cluster size derived by Candel et al. [21] and Van Breukelen et al. [36] for trials with homogeneous clustering varied between $12 \%$ and $25 \%$, where homogeneous clustering means that outcome variances, intraclass correlations, number of clusters and cluster sizes are the same across treatment arms. The simulated relative efficiencies of the present study show that the correction factors for the number of clusters in case of heterogeneous clustering may become somewhat larger, partially because larger coefficients of cluster size variation were considered. A more systematic investigation would be required to explore to what extent smaller cluster sizes and smaller numbers of clusters are responsible for these larger correction factors in the case of heterogeneous clustering. Pending such an investigation, the corrections reported in the current study can be considered conservative corrections that are also safe for larger sample sizes.

The Monte Carlo simulation involved scenarios with three cluster sizes. Our results were checked for other realistic cluster size distributions, involving five and six cluster sizes. Similar to what was found in earlier studies $[21,22]$, the results for these scenarios were in line with the results obtained for the threecluster-size scenarios of the present simulation study.

Finally, randomized trials may involve a binary outcome, such as smoking cessation [27] or offering a follow-up appointment [37], which could be analysed through the logistic mixed effects model. A useful extension of the present study would be the derivation of the ARE and its Taylor approximation for this model, as well as their evaluation in a Monte Carlo simulation study. For cluster randomized trials with homogeneous clustering, this has proven to be a feasible approach [38].

\section{Appendix A: Asymptotic RE for the ML estimator of the treatment effect}

Starting from the linear mixed model in Eqn (1), one can derive, along lines similar to Candel et al. $[22,38]$, the information matrix of the ML-estimators $\hat{\beta}_{0}$ and $\hat{\beta}_{1}$ :

$$
\operatorname{Inf}\left[\begin{array}{c}
\hat{\beta}_{0} \\
\hat{\beta}_{1}
\end{array}\right]=\left[\begin{array}{cc}
\sum_{t} w_{j}^{t}+\sum_{j=K_{t}+1}^{K_{t}+K_{c}} w_{j}^{c} & \sum_{j=1}^{K_{t}} w_{j}^{t} \\
\sum_{j=1}^{K_{t}} w_{j}^{t} & \sum_{j=1}^{K_{t}} w_{j}^{t}
\end{array}\right] \text {, where }
$$


$w_{j}^{t}=\frac{m_{j}^{t}}{m_{j}^{t} \sigma_{u}^{2}+\sigma_{\delta}^{2}}$ and $w_{j}^{c}=\frac{m_{j}^{c}}{m_{j}^{c} \sigma_{v}^{2}+\sigma_{\varepsilon}^{2}}$. The asymptotic variance-covariance matrix is obtained by taking the inverse of (A1), with the entry in the lower diagonal cell giving the variance of $\hat{\beta}_{1}$ :

$$
\operatorname{Var}\left(\hat{\beta}_{1}\right)=\frac{1}{\sum_{j=1}^{K_{t}} w_{j}^{t}}+\frac{1}{\sum_{j=K_{t}+1}^{K_{t}+K_{c}} w_{j}^{c}}
$$

Let $w_{e}^{t}=\frac{\bar{m}^{t}}{\bar{m}^{t} \sigma_{u}^{2}+\sigma_{\delta}^{2}}$ and $w_{e}^{c}=\frac{\bar{m}^{c}}{\bar{m}^{c} \sigma_{v}^{2}+\sigma_{\varepsilon}^{2}}$, with $\bar{m}^{t}$ and $\bar{m}^{c}$ the average cluster sizes in the treatment and control condition, respectively. The ARE of unequal versus equal cluster sizes, keeping the average cluster sizes for both arms constant, can now be written as:

$$
R E\left(\hat{\beta}_{1}\right)=\left(\frac{1}{\sum_{j=K_{t}+1}^{K_{t}+K_{c}} w_{j}^{c}}+\frac{1}{\sum_{j=1}^{K_{t}} w_{j}^{t}}\right)^{-1}\left(\frac{1}{K_{c} w_{e}^{c}}+\frac{1}{K_{t} w_{e}^{t}}\right) .
$$

\section{Appendix B: Optimal restoration of the efficiency loss due to varying cluster sizes}

Let RE denote the relative efficiency of varying versus equal cluster sizes. Assume equal cluster sizes within (but not between) treatment arms, and assume that $K_{t}$ and $K_{c}$ are the cluster numbers in the treatment and control arm that minimize the variance of the treatment effect estimator under the following cost function:

$$
C=C_{0}+K_{t} c_{t}^{*}+K_{c} c_{c}^{*},
$$

where $C_{O}$ are overhead costs and $c_{t}^{*}$ and $c_{c}^{*}$ are the total costs of a treatment and control cluster. To restore the efficiency loss due to varying cluster sizes, we can increase the number of clusters in both arms, that is, take as sample size $f_{t} K_{t}$ and $f_{c} K_{c}$, where $f_{t}$ and $f_{c}$ are the multiplication factors in the treatment and control arm respectively, needed to restore the efficiency loss. From Eqn (A3), these factors should satisfy the following constraints in order to restore the efficiency loss:

$$
\frac{\alpha}{f_{t}}+\frac{\beta}{f_{c}}=R E \times(\alpha+\beta), \text { where } \alpha=\frac{1}{\sum_{j=1}^{K_{t}} w_{j}^{t}} \text { and } \beta=\frac{1}{\sum_{j=K_{t}+1}^{K_{t}+K_{c}} w_{j}^{c}}
$$

This constraint can be rewritten as

$$
f_{t}=\frac{\alpha}{R E \times(\alpha+\beta)-\beta / f_{c}} .
$$

The two factors $f_{t}$ and $f_{c}$, are now chosen such that the cost function in Eqn (B1) is minimized:

$$
C_{0}+f_{t} K_{t} c_{t}^{*}+f_{c} K_{c} c_{c}^{*}=C_{0}+K_{t} c_{t}^{*} \alpha /\left(R E \times(\alpha+\beta)-\beta / f_{c}\right)+f_{c} K_{c} c_{c}^{*} .
$$

Setting the derivative of Eqn (B4) with respect to $f_{c}$ equal to 0 , we obtain

$$
-\frac{K_{t} c_{t}^{*} \alpha \beta}{\left(R E \times(\alpha+\beta)-\beta / f_{c}\right)^{2}} \times \frac{1}{f_{c}^{2}}+K_{c} c_{c}^{*}=0 \Leftrightarrow
$$




$$
\frac{K_{c} c_{c}^{*}}{K_{t} c_{t}^{*}}=\frac{\alpha \beta}{\left(R E \times(\alpha+\beta)-\beta / f_{c}\right)^{2}} \times \frac{1}{f_{c}^{2}} .
$$

The ratio of optimal cluster numbers in case of constant cluster sizes satisfies (Eqn (6) in [13]) $\left(K_{t} / K_{c}\right)^{2}=\left(c_{c}^{*} w_{e}^{c}\right) /\left(c_{t}^{*} w_{e}^{t}\right)$, so that we can rewrite $K_{c} c_{c}^{*} / K_{t} c_{t}^{*}$ in Eqn (B5) as $\gamma\left(\frac{\beta}{\alpha}\right)$, where $\gamma$ is the ARE for the mean outcome in the control arm divided by the ARE for the mean outcome in the treatment arm [19], in formula:

$$
\gamma=\frac{K_{t} w_{e}^{t}}{\sum_{j=1}^{K_{t}} w_{j}^{t}} \times \frac{\sum_{j=K_{t}+1}^{K_{t}+K_{c}} w_{j}^{c}}{K_{c} w_{e}^{c}} .
$$

Substituting this result into Eqn (B5), we obtain, after some rewriting:

$$
f_{c}^{2}[R E \times(\alpha+\beta)]^{2}\left(\frac{\beta}{\alpha}\right) \gamma-2 f_{c} R E \times(\alpha+\beta)\left(\frac{\beta^{2}}{\alpha}\right) \gamma+\beta\left(\left(\frac{\beta^{2}}{\alpha}\right) \gamma-\alpha\right)=0 .
$$

Solving for $f_{c}$ yields two solutions, only one of which is in the domain for $f_{c}$ as defined by Eqn (B3), that is, $[\beta / R E \times(\alpha+\beta), \infty)$, namely

$$
f_{c}^{o p t}=\frac{\alpha+\beta \sqrt{\gamma}}{R E \times(\alpha+\beta) \times \sqrt{\gamma}} \text { and thus } f_{t}^{o p t}=\frac{\alpha+\beta \sqrt{\gamma}}{R E \times(\alpha+\beta)} .
$$

Because the second derivative of Eqn (B4) is positive for $f_{c}$ in Eqn (B8), it minimizes the costs.

For constant cluster sizes, we have $\gamma=1$ and $R E=1$, and so $f_{t}^{o p t}=f_{c}^{o p t}=1$. For varying cluster sizes with the same cluster size distribution and variance parameters in both treatment arms, $\gamma=1$ and so $f_{t}^{o p t}=f_{c}^{o p t}=1 / R E$. In general, $f_{t}^{o p t} / f_{c}^{o p t}=\sqrt{\gamma}$, where $\gamma$ is the relative efficiency for the mean outcome in the control arm divided by the relative efficiency for the mean outcome in the treatment arm [19]. So if $\gamma>1$, the efficiency loss due to cluster size variation in the treatment arm is larger than in the control arm and more clusters should be added to the treatment arm than to the control arm in repairing the efficiency loss. A problem with the costminimizing factors in Eqn (B8) is that they require knowledge on $\alpha, \beta$ and $\gamma$, which mostly will not be available. However, choosing $f_{t}=f_{c}=1 / \mathrm{RE}$ can be shown to be highly efficient, with costs differing from the costs for the optimal replication factors by less than $1 \%$ in almost all cases. A proof of this result is available upon request from the first author.

\section{References}

1. Raudenbush SW. Statistical analysis and optimal design for cluster randomized trials. Psychological Methods 1997; 2:173-185.

2. Roberts C, Roberts SA. The design and analysis of clinical trials with clustering effects due to treatment. Clinical Trials 2005; 2:152-162.

3. Pals SL, Murray DM, Alfano CM, Shadish WR, Hannan PJ, Baker WL. Individually randomized group treatment trials: a critical appraisal of frequently used design and analytic approaches. American Journal of Public Health 2008; 98:1418-1424.

4. Baldwin SA, Stice E, Rohde P. Statistical analysis of group-administered intervention data: reanalysis of two randomized trials. Psychotherapy Research 2008; 18:365-376.

5. Tasca GA, Illing V, Ogrodniczuk JS, Joyce AS. Assessing and adjusting for dependent observations in group treatment research using multilevel models. Group dynamics: Theory, Research and Practice 2009; 13:151-162.

6. Walwyn R, Roberts C. Therapist variation within randomized trials of psychotherapy: implications for precision, internal and external validity. Statistical Methods in Medical Research 2010; 19:291-315.

7. Moerbeek M, Van Breukelen GJP, Berger MPF. Design issues for experiments in multilevel populations. Journal of Educational and Behavioral Statistics 2000; 25:271-284.

8. Liu X. Statistical power and optimum sample allocation ratio for treatment and control having uequal costs per unit of randomization. Journal of Educational and Behavioral Statistics 2003; 28:231-248.

9. Grissom RJ. Heterogeneity of variance in clinical data. Journal of Consulting and Clinical Psychology 2000; 68:155-165. 
10. Manju Md A, Candel MJJM, Berger MPF. Sample size calculation in cost-effectiveness cluster randomized trials: optimal and maximin approaches. Statistics in Medicine 2014; 33:2538-2553.

11. Lix LM, Keselman JC, Keselman HJ. Consequences of assumption violations revisited: a quantitative review of alternatives to the one-way analysis of variance "F" test. Review of Educational Research 1996; 66:579-619.

12. Moser BK, Stevens GR, Watts CL. The two-sample $t$ test versus Satterthwaite's approximate $F$ test. Communications in Statistics: Theory and methods 1989; 18:3963-3975.

13. Candel MJJM, Van Breukelen GJP. Sample size calculation for treatment effects in randomized trials with fixed cluster sizes and heterogeneous intraclass correlations and variances. Statistical Methods in Medical Research 2015; 24(5):557-573.

14. Hox J. Multilevel Analysis. Lawrence Erlbaum: Mahwah, New Jersey, 2002.

15. Feng Z, McLerran D, Grizzle J. A comparison of statistical methods for clustered data analysis with Gaussian error. Statistics in Medicine 1996; 15:1793-1806.

16. Chu R, Thabane L, Ma J, Holbrook A, Pullenayegum E, Devereaux PJ. Comparing methods to estimate treatment effects on a continuous outcome in multicentre randomized controlled trials: A simulation study. BMC Medical Research Methodology 2011; 11:21.

17. Hayes RJ, Moulton LH. Cluster Randomised Trials. CRC Press: Boca Raton, 2009.

18. Eldridge SM, Ashby D, Kerry S. Sample size for cluster randomized trials: effect of coefficient of variation of cluster size and analysis method. International Journal of Epidemiology 2006; 35:1292-1300.

19. Van Breukelen GJP, Candel MJJM, Berger MPF. Relative efficiency of unequal versus equal cluster sizes in cluster randomized and multicentre trials. Statistics in Medicine 2007; 26:2589-2603.

20. Van Breukelen GJP, Candel MJJM. Efficiency loss because of varying cluster size in cluster randomized trials is smaller than literature suggests. Statistics in Medicine 2012; 31:397-400.

21. Candel MJJM, Van Breukelen GJP, Kotova L, Berger MPF. Optimality of equal vs. unequal cluster sizes in multilevel intervention studies: a Monte Carlo study for small sample sizes. Communications in Statistics-Simulation and Computation 2008; 37:222-239.

22. Candel MJJM, Van Breukelen GJP. Varying cluster sizes in trials with clusters on one treatment arm: sample size adjustments when testing treatment effects with linear mixed models. Statistics in Medicine 2009; 28:2307-2324.

23. Manatunga AK, Hudges MG, Chen S. Sample size estimation in cluster randomized studies with varying cluster size. Biometrical Journal 2001; 43:75-86.

24. De Jong K, Moerbeek M, Van der Leeden R. A priori power analysis in longitudinal three-level multilevel models: An example with therapist effects. Psychotherapy Research 2010; 20:273-284.

25. Parker DR, Evangelou E, Eaton CB. Intraclass correlation coefficients for cluster randomized trials in primary care: The cholesterol education and research trial (CEART). Contemporary Clinical Trials 2005; 26:260-267.

26. Kinmonth A-L, Woodcock A, Griffin S, Spiegall N, Campbell MJ. Randomised controlled trial of patient centred care of diabetes in general practice: impact on current wellbeing and future disease risk. British Medical Journal 1998; 317:1202-1208.

27. Herzog TA, Lazev AB, Irvin JE, Juliano LM, Greenbaum PE, Brandon TH. Testing for group membership effects during and after treatment: The example of group therapy for smoking cessation. Behavior Therapy 2002; 33:29-43.

28. Hoover DR. Clinical trials of behavioral interventions with heterogeneous teaching subgroup effects. Statistics in Medicine 2002; 21:1351-1364.

29. Tasca GA, Illing V, Joyce AS, Ogrodniczuk JS. Three-level multilevel growth models for nested change data: a guide of group treatment researchers. Psychotherapy Research 2009; 19:453-461.

30. Jolly K, Bradley F, Sharp S, Smith H, Thompson S, Kinmonth A-L, Mant D. Randomised controlled trial of follow up care in general practice of patients with myocardial infarction and angina: final results of Southampton heart integrated care project (SHIP). British Medical Journal 1999; 318:706-711.

31. Newton-John TRO, Spence SH, Schotte D. Cognitive-behavioral therapy versus EMG feedback in the treatment of chronic low-back-pain. Behaviour Research and Therapy 1995; 33:691-697.

32. Rasbash J, Charlton C, Browne WJ, Healy M, Cameron B. MLwiN Version 2.02. Centre for Multilevel Modelling. University of Bristol: Bristol, 2005.

33. Cohen J. A power primer. Psychological Bulletin 1992; 112:155-159.

34. Mundinger MO, Kane RL, Lenz ER, Totten AM, Tsai WY, Cleary PD, Friedewald WT, Siu AL, Shelanski ML. Primary care outcomes in patients treated by nurse practitioners or physicians. A randomized trial. Journal of the American Medical Association 2000; 283:59-68.

35. Roth A, Rogowski O, Yanay Y, Kehati M, Malov N, Golovner M. Teleconsultation for cardiac patients: A comparison between nurses and physicians. The SHL experience in Israel. Telemedicine and E-health 2006; 12:528-534.

36. Van Breukelen GJP, Candel MJJM. Calculating sample sizes for cluster randomized trials: we can keep it simple and efficient!. Journal of Clinical Epidemiology 2012; 65:1212-1218.

37. Wallace P, Haines A, Harrison R, Barber J, Thompson SG, Jacklin P, Roberts J, Lewis L, Wainwright P. Joint teleconsultations (virtual outreach) versus standard outpatient appointments for patients referred by their general practitioner for a specialist opinion: a randomised trial. Lancet 2002; 359:1961-1968.

38. Candel MJJM, Van Breukelen GJP. Sample size adjustments for varying cluster sizes in cluster randomized trials with binary outcomes analyzed with second-order PQL mixed logistic regression. Statistics in Medicine 2010; 29:1488-1501.

\section{Supporting information}

Additional supporting information may be found in the online version of this article at the publisher's web site. 